Table I shows the action of a number of non-typhoid and typhoid sera upon the B. coli, with the amount of dilution with which the reaction could be obtained. The coli organism used was one which gave in a marked degree the characters of the B. coli, such as indol formation, coagulation of milk, gas formation, marked growth on potato, etc.

TABLE I.

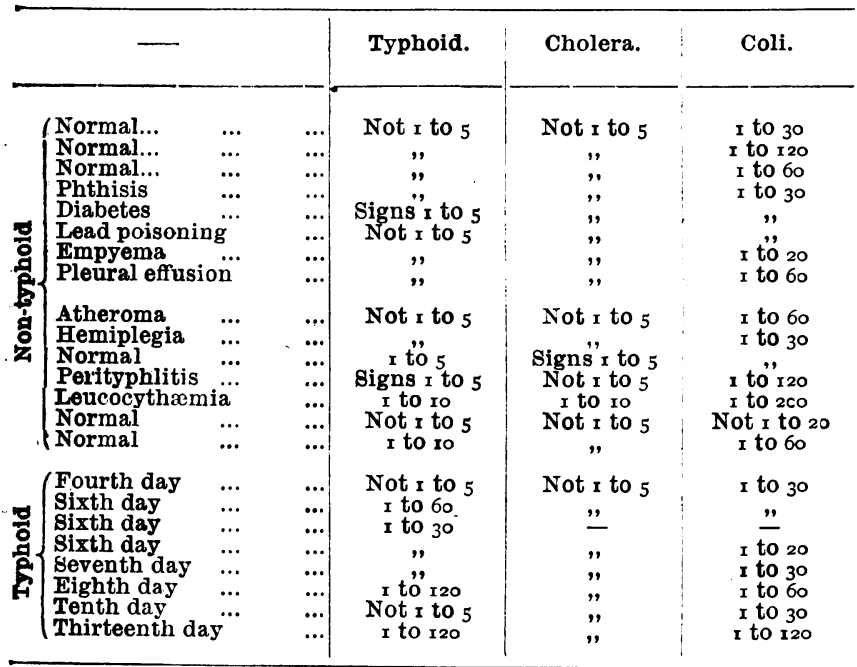

At the dilutions given the action was not pronounced negative until after two hours. At such small dilutions as i to 20 the coli organism was agglutinated within twenty minutes with precisely simila

In crder to see to what extent this action would hold good with different varieties of colon bacillus, I tried the action of normal and typhoid sera in dilution upon 16 cultures of supposed coli organisms taken from different sources. Three of these were acting pathogenically, 4 were from sewage, from milk, 3 from mussels, and I from cheese.

It will be seen that the majority of these were agglutinated by human serum, independently of the fact whether it was from a typhoid patient or not. In fact, had any of these been suspected typhoid organisms, and the action of human typhoid serum been tried upon them, they might very easily have been returned as typhoid bacilli.

TABLE II.

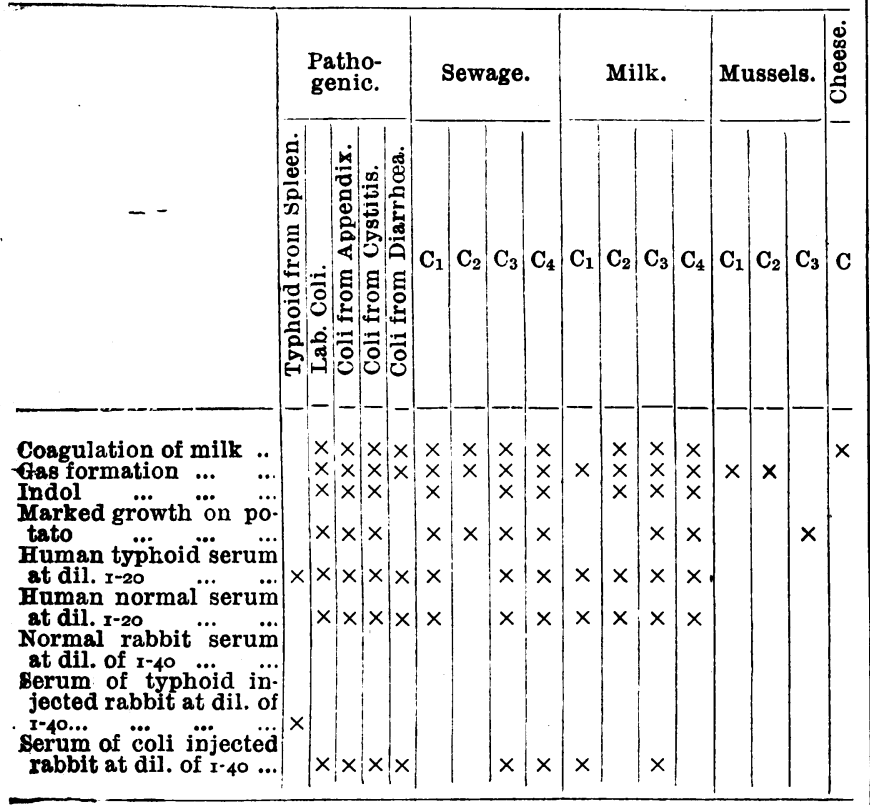

In face of these results $I$ think that in the use of human typhoid serum for diagnosing suspected typhoid bacill found in water, milk, etc., the equally specific action of the serum upon B. coli must also be borne in mind.

In the examination of a number of rabbits' sera, I found that in the majority of cases dilution to the extent of $I$ in 20 did away with all agglutinating action upon both the typhoid and colon bacillus. Such animals injected with typhoid cultures ought (supposing a strict specific action) to give a serum agglutinating the typhoid bacillus and not the colon bacillus.

In Table II it will be seen that on none of the coli-like organisms had the serum of a typhoid rabbit (in dilution) any action, whilst it had a marked action on typhoid bacilli. So that presumably the results of those observers who have used the sera of injected animals as the differentiating agent have escaped the above-mentioned error.

However, even if injected animals be used, the serum ought to be proved to have no action upon the colon bacillus in the dilution at which it is proposed to use it as a differentiating test.

\section{PRACTICAL SUGGESTIONS} ON THE

\section{TREATMENT OF STRICTURE OF THE URETHRA.*} BY JOHN WARD COUSINS, M.D., F.R.C.S.,

Senior Surgeon to the Royal Portsmouth Hospital, and to the Portsmouth and South Hants Eye and Ear Infirmary.

STRICTURE of the urethra may occur at any age from tuberculous disease or injury, but in a vast proportion of cases gonorrhœa is the primary cause. Living in the midst of a large seafaring population, we have abundant opportunities of treating every variety of urethral disorder. Bailors, as a class, live careless lives, and the diseases which they contract are too often treated by ignorant and unqualified persons. Injections are employed in all stages of specifio urethral inflammation without any precautions at all, and their careless application, together with the neglect of local cleanliness, promotes the extension of the disease along the mucous surface of the canal.

During the last few years my views on the treatment of urethral stricture have undergone very considerable modification. I have long given up all attempts at forcible rupture, and likewise the operation of internal urethrotomy. Before the latter operation can be practised the strictured part must be dilated to admit the cutting instrument, and a sound equivalent to a No. 2 or 3 catheter must be successfully passed along the canal. Apart, therefore, from all other objections to this method, I am confident there are very few cases which cannot be cured by dilatation whenever an instrument of this size has been introduced into the bladder.

Dilatation UNDER an AN anstheric.

It is my experience that the tightest and thickest strictures in any part of the canal can generally be cured by simple intermittent dilatation; and for the practice of this operation many kinds of instruments have been introduced from time to time, so that it would be a difficult task to develop any very startling improvement.

The special instruments which I employ are tapering catheters, with bulbous points and solid flexible beaks. The orifice of the tube is placed at the base of the beak, and the point of the straight stilette exactly fits the bore of the instrument (Fig. 1).

A tapering metallic instrument presents many advantages in the treatment by dilatation: (1) It enables the surgeon to rapidly pass several sizes of dilators at one sitting; (2) the flexible beak is serviceable in changing the direction of the point, which is a little manœuvre often of great advantage in penetrating a tortuous stricture; (3) the bulbous extremity assists the operator in determining that penetration has been successfully accomplished.

It is always a good plan to delay the operation of dilatation until the patient has been prepared by rest, regular and un-

* Read in the Section of Surgery at the Annual Meeting of the British Medical Association held at Montreal in September, 1897. 
stimulating diet, 'and general remedies. The preparation of the patient is an essential part of the treatment, for these precautionary measures, when carried out for a few days, render surgical interference more easy, and also tend to reduce the irritability of the canal. can carry it out, and completely deodorise a set of catheters in half an hour. The steaming apparatus and the tapering catheters have been made for me by Messrs. Arnold and Sons.

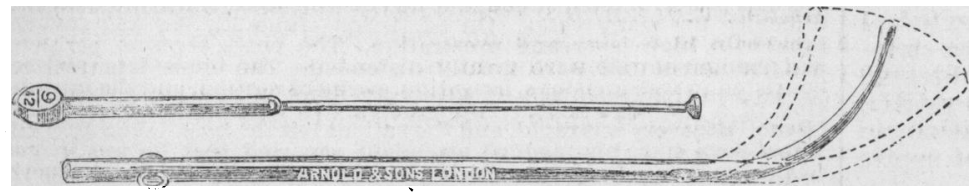

FIg. I, $\rightarrow$ A tapering metallic catheter with flexible beak and straight stilette.

It is a matter of common experience that the urinary organs present very varying degrees of irritability. Some patients are able to bear dilatation without any inconvenience, while others suffer great local irritation, attended with fever and other urgent symptoms. The passage of an instrument in a healthy man has occasionally excited dangerous constitutional disturbance. I have frequently observed that patients who have suffered from malarial poisoning in foreign climes are markedly predisposed to urinary fever. With them especially surgical interference with the urino-genital system has a tendency to produce febrile trouble, and the nervous currents which are thus excited appear to radiate widely through the body, and to rouse through old channels a latent irritability of the nervous system.

:Sterilised Instruments for the Dilatation of Urethrad STRICTURE.

The absolute necessity for cleaning and deodorising catheters in the treatment of stricture is now universally admitted, but in my experience the process is often very imperfectly carried out. I have seen many cases of nrinary abscess which could be fairly traced to the habitual use of old and dirty instruments. Now the cleaning process which I employ in my practice consists of two stages : (1) A steaming process, and (2) polishing the interior of the tubes.

The apparatus consists of a boiler having a capacity of about 3 pints, and capable of bearing a pressure of $80 \mathrm{lbs}$. to the square inch. It is heated by a spirit lamp. The steam pipe is connected with a vertical tube fitted with projecting terminals, and these are adapted to hold a complete set of catheters. Steam cocks are arranged on the steam pipe to regulate the discharge of steam, and $a$ tray is placed beneath the instruments to collect the condensed water. I have converted a disused steam spray into a catheter deodoriser. The apparatus is represented in the woodcut. After steaming

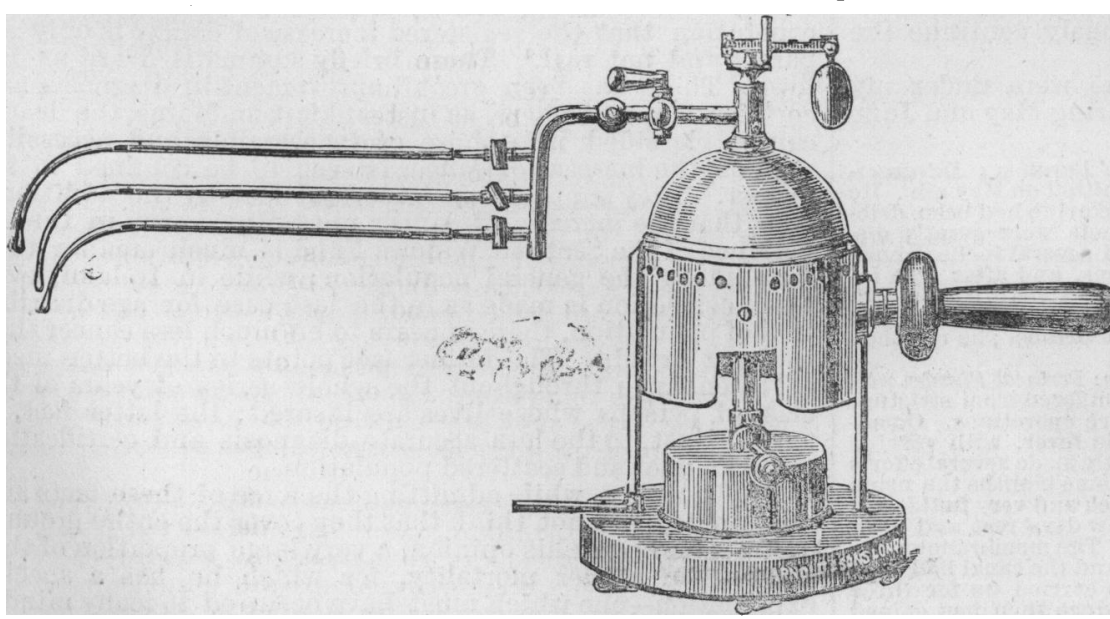

Fig. 2.-The catheter deodoriser.

For three or four minutes, the instruments are wiped dry with a soft leather, and their insides polished with the steel stilettes. The point of each stilette consists of a flat metal disc which exectly fits the bore of the instrument. By the friction of the disc, the interior of the tube can be polished in one minute. The process is very simple, so that any nurse
Treatment of Chronic ann Impassable Stricture. The operation of rapid dilatation is not suitable for many clironic cases, and their treatment has to be conducted with the greatest caution. Strictures are often followed by changes in the urethra between the meatus and the injured part of the canal, involving excoriation, ulceration, or even perforation of the mucous surface. Behind the stricture the walls are dilated and pouched, and the capacity of the bladder is reduced by slow thickening of the mucous and muscular coats. The ureters and the pelves of the kidneys, too, are often involved in the changes, which ('xtend along the urinary tract, and these organs themselves become softened and attacked with serious interstitial disease. Hence in all cases of old stricture the treatment must be conducted in carefully regulated stages, and the urine submitted to frequent examination. It is often desirable to estimate the average amount of urea contained in the secretion, for in every case the danger of surgical treatment is in direct propostion to the extent of the renal degeneration.

In some cases in which the introduction of any instrument has been attended with very considerable difficulty, I have used with much satisfaction a capillary catheter, and this perfectly flexible little instrument can really do injury. It is a compound contrivance consisting of a filiform bougie and a fine catheter, very carefully prepared with woven web and gum elastic, and possesses great flexibility and tougbness, together with a smooth and highly polished surface. The combination is about 18 inches in length. ${ }^{1}$

With reference to the method of using the capillary catheter, it must always be handled with great delicacy and patience. After injecting the urethra with warm oil, the penis is drawn forward with the left hand, and the instrument gently passed down to the stricture. As soon as its progress is arrested it must be withdrawn two or three inches, rotated between the thumb and finger, and again twisted down upon the obstruction. During the repetition of these manipulations it often slips into the bladder, at the same time its free and easy movement in the urethra, toge her with the sensations experienced by the patient, clearly indicate to the operator that the stricture has been overcome, and the catheter coaxed to move on in the right direction. I generally retain the instrument in position for about thirtygenerally

The operation of dilatation after prolonged rest and many patient efforts, sometimes proves unsuccessful, and the patient remains exposed to the risk of many terrible complications. Under these circumstances I always recommend the operation of external urethrotomy, followed by bladder drainage through the perineam, as the very best surgical procedure that can be practised.

Some surgeons have asserted that, if urine can be discharged through the canal, it ought to be possible by skill and perseverance for an instrument of some kind to be introduced through the stricture into the bladder. I cannot, however, admit this statement, for the urethra may be absolutely impermeable to instruments, although the urine can be discharged in a tiny and tortuous stream.

In all cases, then, of chronic and obstinate stricture, when other methods have fairly failed, and in cases of extravasarion of urine, it is my practice to perform external urethrotomy in the manner suggested by $\mathbf{M r}$. Wheelhouse, and I believe this operation will supersede all the old dodges of finding a way into the bladder without a guide. After the operation the evacuation of the urine is carried on through the perineal wound for some days by means of en india-rubber tube. Perineal drain- 
age secures the advantage of diverting the stream of urine, and also of permitting the anterior part of the passage to be effectually deodorised and kept at resta very important expedient for preventing the development of that form of febrile disturbance which is intimately associated with the contact of disordered urine with a wounded surface. Moreover, in cases of external urethrotomy for the rtlief of obstinate and unyielding strictures, the structures of the perineum are already infiltrated and unhealthy, the bladder is the seat of chronic cystitis, and the urine is persistently in a state of decomposition, so that under these conditions there is a special risk of septic absorption.

In all cases of extravasation of urine direct drainage through the perineum is absolutely necessary, as it is the only channel of escape for the urine well away from the swollen and sloughing tissues. Depend upon it, the most heroic incisions will prove insufficient unless the urethra is freely opened and a large drainage tube introduced just within the bladder. The operation can be very conveniently completed in the following stages: (1) Ether having been administered, and the patient placed in the lithotomy position, free incisions must be made wherever the urine appears to have penetrated, and the imprisoned fluids squeezed out with the hand-a manipulation which is much assisted hy hot antiseptic irrigation. (2) Perineal section, followed by complete division of the strictured parts of the canal should now be performed. When it is possible to pass a staff through the contract $\epsilon d$ urethra into the bladder, this part of the operation is converted into a very simple proceeding but when this cannot be accomplished the urethra must be found and the bladder reached without a guide. When re. tention of urine is present the distended passage is readily opened. If, however, the bladder is empty a little patience may be necessary to eneure success. Sometimes I have found the orifice of the tube by the aid of a small probe, and then slipping by its side a blunt pointed director, the stricture has been carefully divided and a urethral gorget passed into the bladder. If any contraction exists in the penile portion of the canal, I divide this subcutaneously through the perineal wound on a straight staff with a narrow and blunt pointed knife. (3) After warm irrigation the operation is completed by introducing a large catheter through the entire length of the urethra, over the gorget into the bladder, and on withdrawing the instrument a drainage tube is inserted through the perineal wound.

The tendency to contraction of the cicatricial tissue in the walls of the canal after dilatation or external urethrotomy may be overcome by the judicious use of dilators. My patients are always taught to introduce a No. 1 I or 12 tapering catheter, and they are urged to assiduously continue the treatment

The following cases of chronic stricture were under my care at the Royal Portsmouth Hospital during May and June of 1897 :

CAsR I. Stricture of the Urethra of Twenty five Years' Duration : Extravasation of Urine.-G. H., aged 57, a carpenter, was admitted on May th. He was very ill, with high fever and rapid pulse. The urine had been dribbling away for some days. The scrntum and penis were greatly disthe umbilicus. He was at once placed nnder ether, and after free incisions perineal section was performed, and two strictures divided. Drainage was continued for two weeks. He made rapid progress, and soon learnt to pass a No. I catheter. Last seen in October; he was then in good health and able to follow his employment.

CASE II. Stricture of the Urethra Tuenty-seven Years: Perineal Fistulx, with Fotid Cystitis.-B. B, aged 52, stated that he had suffered from stricture twenty-seven sears, and had undergone two severe operations. On admission, May 7 th, he was labouring under septic fever, with varying tomperature and greati prostration. He had recently made several efforts to introduce a snall gum elastic catheter, as for some months the urine had escaped from the fistulæ. The urine was thick and very fotid, and the perineum was liard and swollen. After a few days' rest and antition of the urethra was found almnst nbliterated. and the canal had to be tion of the urethra was found almnst obliterated. and the canal had to be weeks. He left the hospital on June 9 th, and since then has gained olbs. in weight, He continues to pass a No. 12 tapering catheter three imes a week. The perineum is quite sound, and the fistulm have healed. CASE III. Chronic Stricture, with Acute Urinary Fever and Extravasation. -H. J., aged 54, a shipwrjght, was admitted on May 25 th. He stated that he had suffered from stricture many years, and had been in the habit of passing a small catheter. The penis was much, swollen with brawny hardness around the base and in both snermatic regions. The urine was dis through the penile stricture. After free incision and irrigation, perineal section was performed, two strictures divided. and a drainage tube passed into the bladder. The inguinal regions were also drained through the scrotal incisions. Progress was slow, and the drainage and antiseptic treatment had to be continued for three weeks. He left the hnspital on July roth, capable of passing easily a No. 12 tapering catheter. He is now in good health, and has gained many pounds in weight.

CASE IV. Chronic Stricture and Extravasation of Urine.-H. D., aged 50 , a tall and powerful man, stated that he had evacuated his urine with great difficulty for many years. On admission-June 6th-he was suffering great pain, high fever, and prostration. The penis, scrotum, perineum, and abdominal wall were greatty distended. The immediate treatment consisted in free incisions, irrigation. perineal section, and the introduction of a drainage tube. The urethra was surrounded by sloughing tissue. Recovery was rapid, and the patient left the hospital on July irth. A few days since his medical attendant reported that he was in good
health, and regularly introducing without difficulty a No. II tapering health, and
catheter.

REFERENCE.

1 BRITISH Medical JoURNAL, December rgth, 1895

\section{AN EXPLANATION OF THE SUPPOSED INCREASE OF CANCER MORTALITY IN THIS COUNTRY.}

\author{
By ARTHUR NEWSHOLME, M.D.LoND., M.R.C.P., \\ Medical Officer of Health, Brighton.
}

In a recent paper read before the members of the Insurance Association of Manchester, Mr. W'm. Thorburn, F.R.C.S., enters into an interesting discussion of this subject, and the following remarks consist chiefly of a criticism of the facts and inferences contained in this paper. Mr. Thorburn first of all draws attention, as all other writers on the subject have done, to the immense increase of proportional mortality registered as having been caused by "malignant disease," the proportion of deaths from malignant disease to total deaths from all causes having risen from $I$ in 74 in 1854 , to $I$ in 23 in 1894. This increase, if established as an actual fact, has an important bearing on the work of insurance offices; because, as Mr. Thorburn point out, (a) the increase is especially among males, who form the vast majority of the assured ; (b) cancer comes on after the age at which insurance is usually effected ; and $(c)$ it occurs to a predominant extent in those of good social position, who are most likely to insure their lives. This statement of the case we may mention in passing is only correct in so far as the increased amount of cancer causes the mortality among the insured to occur at an earlier age than would occur in its absence. All must die ; when cancer causes premature death, and only then, can it be said to have an unfavourable effect upon the practice of insurance offices.

Mr. Thorburn then summarises some of the considerations which led Mr. King, F.I.A., and the present writer to the conclusion that the registered increase of cancer is only apparent and not real. ${ }^{1}$ These briefly summarised are as follows: There has been great improvement in diagnosis and certification, and where, as in Frankfort-on-Maine, the deaths can be classified into those of inaccessible and accessible regions, the increase of cancer is seen to be confined to the former. They support this important fact by the additional facts that the increase of cancer mortality shown in the experience of the Scottish Widows Fund is much smaller than that among the general population; while in Ireland, even when correction is made as in the last case for age distribution of population, there appears to be much less cancer than in Great Britain. The former fact points to the better medical attendance throughout the whole series of years of the class of persons whose lives are insured; the latter fact, in part at least, to the less accurate diagnosis and certification among a poor and scattered population.

Mr. Thorburn, while admitting the force of these facts and arguments, does not think that they cover the entire ground. There remains, in his opinion, a very large proportion of this increase of cancer mortality, for which he has a special explanation-one which must have occurred to many minds. Mr. Thorburn has evidently failed to appreciate the fact that Mr. King and the present writer have entirely eliminated the cause of disturbance of death-rates, on which his explanation is based, by a novel method of correction for age and sex distribution of population, which is fully described and illustrated in their paper. ${ }^{2}$

Mr. Thorburn's explanation is that with the lowered 